\title{
Treatment with clobetasol propionate $0.025 \%$ topical therapy in various dermatoses
}

\author{
Ashwini Guttedar ${ }^{1 *}$, Nitin Mishra², Sanjay Kumar Gupta ${ }^{3}$, Atul Dilip Mohankar ${ }^{4}$, \\ Karan Sancheti $^{5}$, Kanchan Srivastava ${ }^{6}$, Puja Sharma ${ }^{7}$, Ashok Gangwar ${ }^{8}$, Apoorva Jain ${ }^{9}$, \\ Lakshmi Sitara Ganisetti ${ }^{10}$
}

\begin{abstract}
${ }^{1}$ Department of Dermatology, Derma Care and cosmetic clinic, Warangal, Telangana, India
${ }^{2}$ Department of Dermatology, Dr Nitin Mishra skin clinic and laser centre, Bareilly, Uttar Pradesh, India

${ }^{3}$ Department of Dermatology, Noble Medical Centre, Hyderabad, Telangana, India

${ }^{4}$ Department of Dermatology, Chhattisgarh Institute of Medical Sciences, Bilaspur, Chhattisgarh, India

${ }^{5}$ Department of Dermatology, NRG Polyclinic, Kolkata, West Bengal, India

${ }^{6}$ Department of Dermatology, The Doctor's Arena, Lucknow, Uttar Pradesh, India

${ }^{7}$ Department of Dermatology, TT Hospital, Kota, Rajasthan, India

${ }^{8}$ Department of Dermatology, Gangwar Skin \& Fue Hair Transplant Center, Raipur, Chhattisgarh, India

${ }^{9}$ Department of Dermatology, MAX Skin Care Centre, Bhopal, Madhya Pradesh, India

${ }^{10}$ Department of Dermatology, Advanced Skin and Hair Clinic, Hyderabad, Telangana, India
\end{abstract}

Received: 06 December 2021

Accepted: 21 December 2021

\section{*Correspondence:}

Dr. Ashwini Guttedar,

E-mail: ashwiniguttedar14@gmail.com

Copyright: (c) the author(s), publisher and licensee Medip Academy. This is an open-access article distributed under the terms of the Creative Commons Attribution Non-Commercial License, which permits unrestricted non-commercial use, distribution, and reproduction in any medium, provided the original work is properly cited.

\begin{abstract}
Owing to their anti-inflammatory and vasoconstrictive properties. Topical corticosteroids (TCs) provide benefits in various dermatological conditions, including atopic eczema, psoriasis, chronic hand eczema, and localized vitiligo. Clobetasol propionate $(\mathrm{CP})$ is the most common topical agent possessing anti-inflammatory, antimitotic, antipruritic, and immunosuppressive properties that are employed in the management of plaque psoriasis. CP $0.025 \%$ cream was approved by the United States food and drug administration for the treatment of moderate-to-severe psoriasis in adult patients. The formulation is free from known contact allergens, such as propylene glycol, short-chain alcohols, and sorbitol-based emulsifiers, and has demonstrated hypoallergenic effects. High penetration of active ingredients and a lower degree of systemic absorption make CP $0.025 \%$ an effective and safe agent. This case series discusses the clinical experience of using CP $0.025 \%$ cream in various dermatologic conditions, focusing on its efficacy and safety.
\end{abstract}

Keywords: CP $0.025 \%$, Plaque psoriasis, Psoriasis, TCs

\section{INTRODUCTION}

In the last seven decades, TCs have been the mainstay of treatment for several dermatological conditions associated with inflammation and pruritus and characterized by dry, scaly, crusted, or erythematous skin. The management of various skin conditions, such as psoriasis, atopic dermatitis, seborrheic dermatitis, contact dermatitis, and other inflammatory dermatoses, have employed TCs for their diverse biologic properties including anti-inflammatory properties, reduction in neutrophil and monocyte recruitment, stabilization of cellular and lysosomal membranes, and vasoconstrictive properties. $^{1}$

Globally, CP is the most common tropical agent used for the management of psoriasis. ${ }^{2} \mathrm{CP}$ is a fifth-generation corticosteroid and is considered the most potent topical steroid. ${ }^{3}$ The new CP $0.025 \%$ cream formulation has been approved to be applied twice daily for the treatment of 
moderate-to-severe psoriasis in adult patients. CP $0.025 \%$ cream does not contain known contact allergens, such as propylene glycol, short-chain alcohols, and sorbitol-based emulsifiers, unlike the previous CP formulations $(0.05 \%)$. Furthermore, CP $0.025 \%$ cream formulation is associated with lower CP serum levels and less hypothalamicpituitary-adrenal axis suppression compared to the previous formulations. ${ }^{1}$

The present case series discusses the clinical experience of using CP $0.025 \%$ in terms of its safety and efficacy in various dermatological conditions.

\section{CASE SERIES}

\section{Case 1: Efficacy of Impoyz ${ }^{T M} 0.025 \%$ cream in psoriasis lesion localized on elbow: a case report}

A 30-year-old male presented with a single, scaly patch over the elbow. There was no other comorbidity or any significant family history. The vitals and systemic findings were within normal limits, and the patient had no psychological and social sequelae. The lesion of around $3 \times 4 \mathrm{~mm}$ was located on the elbow. The lesion was accompanied by inflammation. Itching and scaling were also noted (Figure $1 \mathrm{~A}$ ). The patient was diagnosed with plaque psoriasis.

Topical Impoyz ${ }^{\mathrm{TM}}(\mathrm{CP})$ cream $0.025 \%$ was prescribed to be applied to the affected skin area at night for 15 days. A moisturizing cream was suggested to be applied in the morning after taking shower. At the follow-up, erythema and plaque elevation had reduced substantially (Figure 1 B). The itching had almost disappeared, providing relief to the patient. Overall, the disease severity had reduced after the treatment.

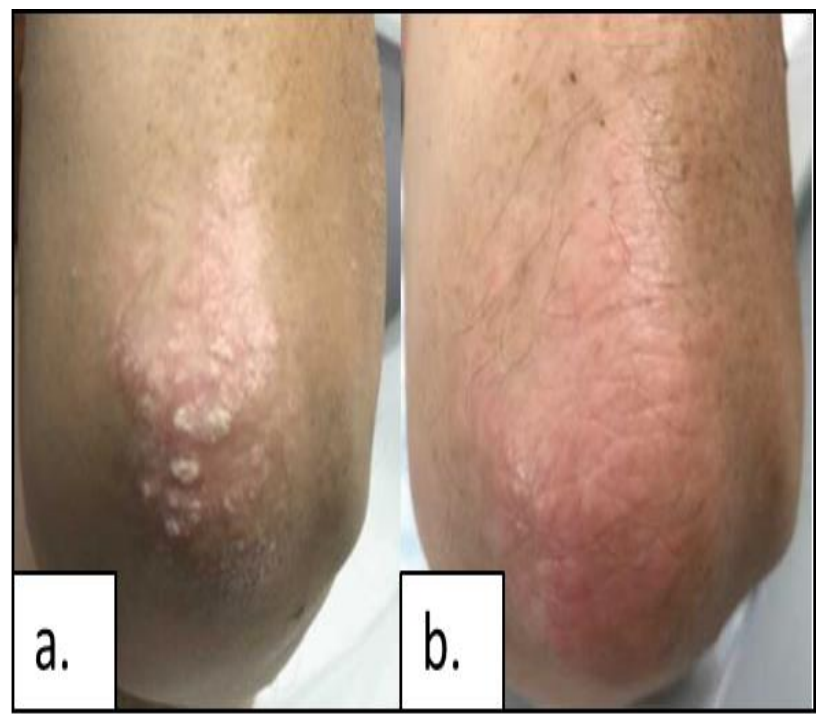

Figure 1 ( $a$ and $b$ ): Pretreatment image of the lesion located on the elbow. Post-treatment image of the lesion of improvement.

\section{Expert opinion}

In this case, the application of $\operatorname{Impoyz}^{\mathrm{TM}}(\mathrm{CP})$ cream $0.025 \%$ resulted in a notable reduction in disease severity and complete itch control. Impoyz ${ }^{\mathrm{TM}}(\mathrm{CP})$ cream $0.025 \%$ can be considered as a first-line treatment for managing patients with psoriasis, considering its efficacy and safety profile. It shows better results when used with moisturizers.

\section{Case 2: Relief from psoriasis lesions on face and neck with Impoyz ${ }^{T M} 0.025 \%$ cream: a case report}

A 21-year-old male presented with scaly eruptions all over the body for the past 2 months. There was no comorbidity or any significant family history. On physical examination, his vitals were found within normal limits. His systemic findings were unremarkable and had no psychological and social sequelae. The lesions were present mainly on the neck and face. Inflammation around the lesions was present. The center of the lesions appeared dry and scaly. Rashes were present with scaling and itching. The affected skin was warm. Around 5\% of the body surface area was affected (Figure $2 \mathrm{~A}$ ). The patient was diagnosed with plaque psoriasis and was prescribed topical Impoyz ${ }^{\mathrm{TM}}$ (CP) cream $0.025 \%$ to be applied to the affected skin areas once daily for 14 days.

At follow-up, improvement in scaling and erythema had been noted. Plaque elevation had completely cured (Figure 2 B). The itching had disappeared completely. Overall, the disease severity had reduced during the treatment period.

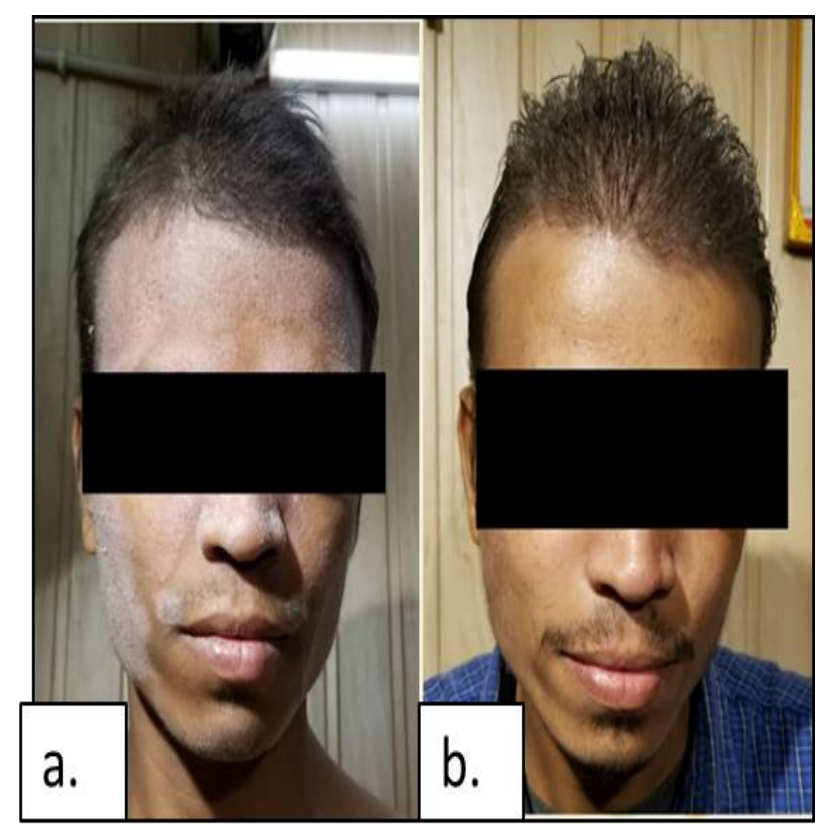

Figure 2 (a and b): Pretreatment image of the dry and scaly skin patches on the face. Post-treatment image of the cured patches indicating improvement. 


\section{Expert opinion}

Impoyz $^{\mathrm{TM}}(\mathrm{CP})$ cream $0.025 \%$ is a safe treatment when applied all over the body. It may be a preferred choice, especially in treating psoriasis in children. Treatment results with $\mathrm{CP}$ cream $0.025 \%$ were satisfactory to the patient as well as the physician.

\section{Case 3: Effective management of flaky and itchy patches of psoriasis with CP $0.025 \%$ cream}

A 37-year-old male presented with flaky patches on the skin accompanied by itching. There was no significant comorbidity or any family history. The physical and systemic findings were unremarkable, and psychological and social sequelae were absent. The lesions were present below the knee. The size of the lesions was around 6 inches. Inflammation around the lesions was present. A rash was present with scaling and itching (Figure $3 \mathrm{~A}$ ). The affected area was warm.

The patient was diagnosed with psoriasis. Topical Impoyz $^{\mathrm{TM}}$ (CP) cream $0.025 \%$ was prescribed to be applied to the affected skin area twice daily for 14 days. The cream was advised to be applied after bath in the morning and at night.

On day 14, when the patient arrived for follow-up, it was observed that scaling and plaque elevation had substantially reduced (Figure 3 B). A substantial improvement in erythema and itching had been noted. There was a notable reduction in flares and relapses. Overall, the disease severity had decreased during the treatment period.

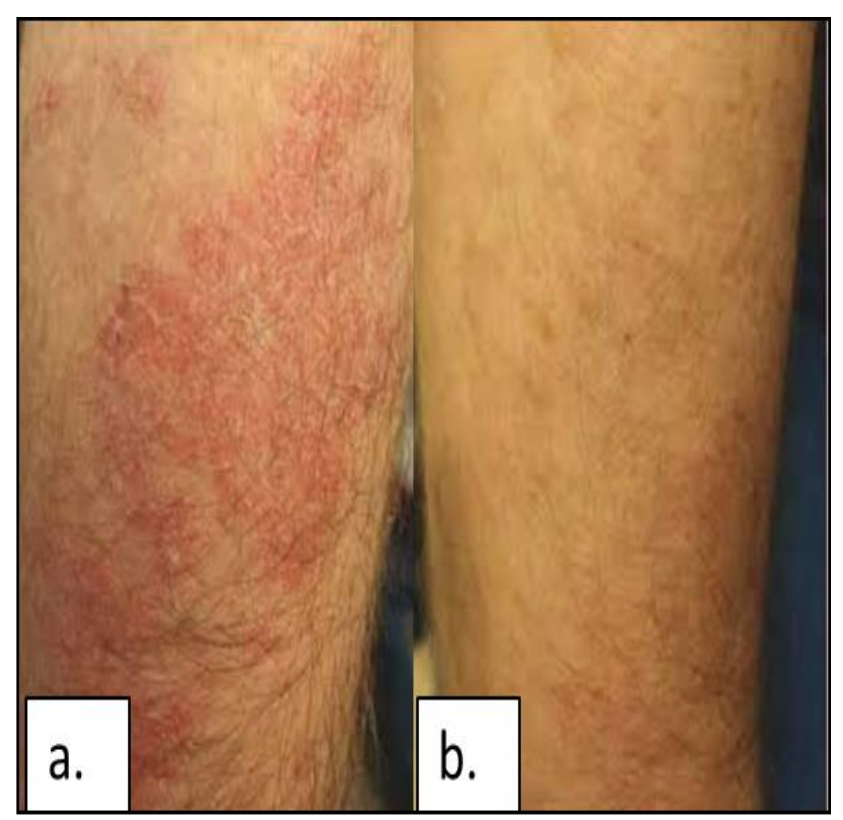

Figure 3 (a and b): Pretreatment image of the lesion on the knee. Post-treatment image of the improved lesions.

\section{Expert opinion}

Impoyz $^{\mathrm{TM}}(\mathrm{CP})$ cream $0.025 \%$ can be recommended as a first-line topical therapy in the management of psoriasis. CP cream $0.025 \%$ is effective and not associated with side effects, including complaints of discoloration.

\section{Case 4: A case of localized plaque psoriasis successfully managed with CP $0.025 \%$}

A 50-year-old male presented with skin thickening and scaling over the sole for the past 2 months. He had difficulty wearing footwear due to the severe itching that he had been experiencing at the site of the lesion (Figure 4 A). There was no other comorbidity or any significant family history. The examination revealed unremarkable vitals and systemic findings, while no psychological and social sequelae were found.

The patient was diagnosed with plaque psoriasis and was prescribed topical Impoyz ${ }^{\mathrm{TM}}(\mathrm{CP})$ cream $0.025 \%$ to be applied to the affected skin area twice daily for 14 days.

The patient showed a decrease in scaling and a reduced erythema and plaque elevation (Figure $4 \mathrm{~B}$ ). He reported that the itch had almost vanished. Overall, the disease severity had improved. The patient was asked to continue with the treatment for 1 more week.

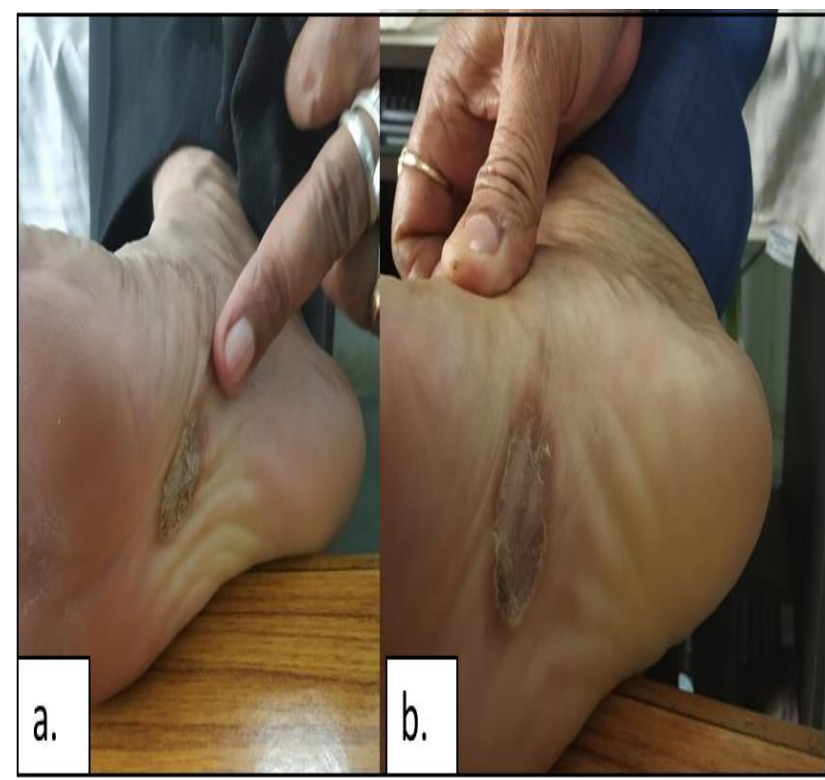

Figure 4 (a and b): Pretreatment image of the lesion on the sole. Post-treatment image of the improved scaling.

\section{Expert opinion}

Application of Impoyz ${ }^{\mathrm{TM}}(\mathrm{CP})$ cream $0.025 \%$ results in an improvement in plaque psoriasis. Due to minimal systemic absorption of CP $0.025 \%$ cream, it is not associated with side effects. 
Case 5: Improvement in palmar psoriasis with CP $0.025 \%$ : a case report

A 55-year-old male presented with scaling and dryness with itching over his palms. He had been diagnosed with hypertension 2 years before and had been taking medications. There was no relevant family history. Both physical and systemic examinations revealed unremarkable findings. There were no psychological and social sequelae. The lesion was located on the palm of both upper limbs. There was no inflammation around the lesion. The center of the lesion was scaly and plaques were observed. Itching and scaling were observed (Figure $5 \mathrm{~A})$. The patient was diagnosed with plaque psoriasis.

The condition was managed with topical Impoyz ${ }^{\mathrm{TM}}(\mathrm{CP})$ cream $0.025 \%$ that was prescribed to be applied to the affected skin area twice daily for 3 weeks. A moisturizer cream was advised to be applied to the skin twice daily for 3 weeks. At the end of week 3, at follow-up, plaque elevation and scaling had reduced substantially (Figure 5 B). The itching had decreased. Overall, the disease severity had improved by more than $80 \%$ after the treatment.

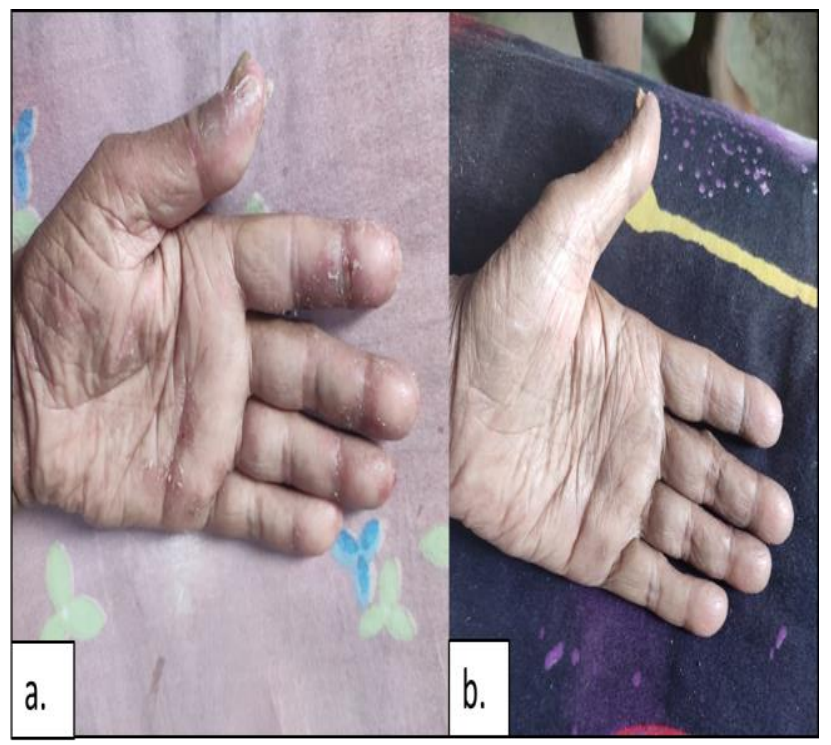

Figure 5 (a and b): Pretreatment image of the lesions of palmar psoriasis. Post-treatment image of the improved scaling.

\section{Expert opinion}

Impoyz $\mathrm{z}^{\mathrm{TM}}(\mathrm{CP})$ cream $0.025 \%$ is efficacious in psoriasis management and can be considered as a first-line treatment especially in the case of palmar psoriasis. It does not lead to any of the side effects that are experienced with other TCs.

Case 6: Efficacy of topical Impoyz ${ }^{T M}$ cream $0.025 \%$ in psoriasis in a female patient with diabetes and hypertension
A 59-year-old female presented with dry, scaly rashes on palms and fingers with cuts and fissures for the past 6 months. She had been a patient of diabetes mellitus for the past 6 years and had been on antidiabetic medications. She had also been diagnosed with hypertension 4 years before and had been taking antihypertensives. She was a housewife taking care of household cleaning and was exposed to detergents and bleaches.

On examination, her vitals and systemic findings were within normal limits. She had no psychological and social sequelae. The lesion was located on both the palms and sides of the fingers. The lesion was present almost on the whole palm and on 3-4 fingers. Inflammation around the lesion was present. The center of the lesion was flaky with fissures. Flaky rash with itching and scaling was noted. Mild tenderness at the lesion site was observed. Around 2\% of body surface area was affected (Figure 6 A).

The patient was diagnosed with psoriasis. She was prescribed topical Impoyz ${ }^{\mathrm{TM}}(\mathrm{CP})$ cream $0.025 \%$ to be applied to the affected skin area twice daily for 14 days. After 14 days, the patient was advised to apply Impoyz ${ }^{\mathrm{TM}}$ (CP) cream $0.025 \%$ once daily for the next 14 days. She was advised to use a moisturizer and mild, nonsoap cleansers. She was instructed to avoid the use of detergents and bleaches during the treatment period.

On the next follow-up, it was seen that the scaling had reduced by almost $80 \%$. Erythema had disappeared and itch had reduced by $90 \%$. There was a reduction in plaque elevation (Figure $6 \mathrm{~B}$ ). Overall, the disease severity had reduced after the treatment.

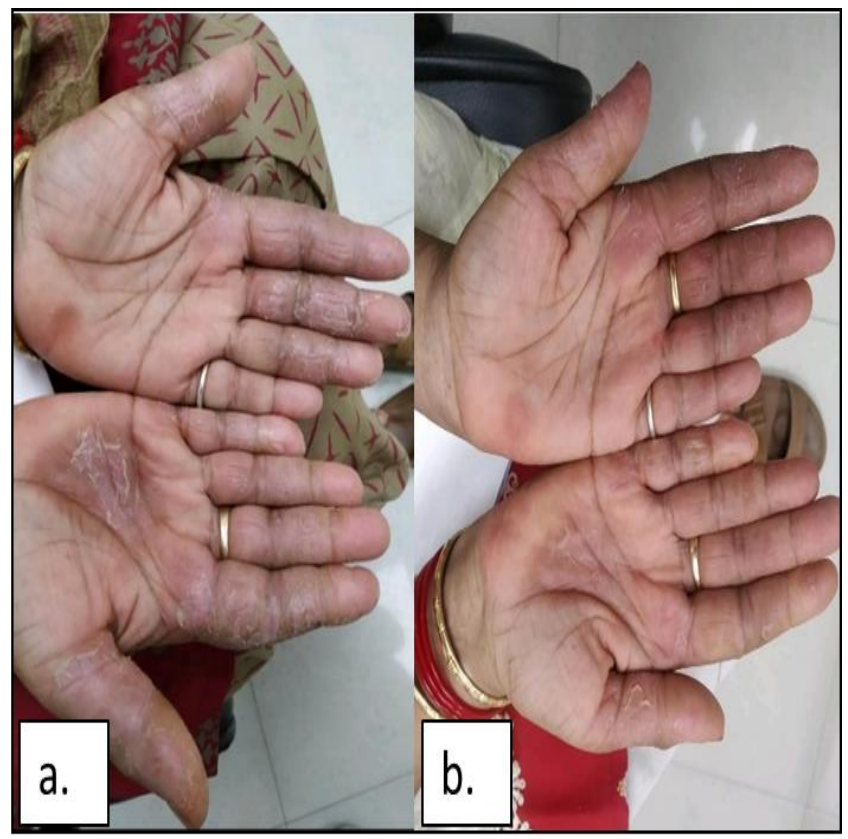

Figure 6 (a and b): Pretreatment image of the lesion on the palm. Post-treatment image of the improved symptoms. 


\section{Expert opinion}

Impoyz ${ }^{\mathrm{TM}}(\mathrm{CP})$ cream $0.025 \%$ is not associated with side effects unlike clobetasol $0.05 \%$. Its ease of prescription and proven efficacy make it a favorable choice in psoriasis management.

\section{Case 7: A case with multiple psoriatic lesions on the extremities managed with CP $0.025 \%$}

A 60-year-old male presented with multiple well-defined plaques with scaling and pigmentation on the extremities. He had been a known case of hypertension for the past 10 years and had been taking antihypertensive medications. There was no family history of a similar dermatological condition.

His physical and systemic examinations revealed no abnormality. His psychological and social reaction was also unaffected. The lesions were located on the thighs and legs of size $10 \times 10 \mathrm{~cm}$ and $10 \times 12 \mathrm{~cm}$. Inflammation around the lesion was present. The center of the lesion was scaly. Itching and scaling were observed (Figure 7 A).

The patient was diagnosed with plaque psoriasis. He was instructed to apply topical Impoyz ${ }^{\mathrm{TM}}(\mathrm{CP})$ cream $0.025 \%$ to the affected skin area twice daily for 20 days. Furthermore, the patient was advised to keep applying oil for adequate skin hydration.

At follow-up, the scaling was noted to have reduced substantially and appeared thin after treatment. Erythema and plaque decreased by almost $50 \%$ (Figure $7 \mathrm{~B}$ ). Itching almost disappeared, providing relief to the patient. Flare-ups or relapse decreased.

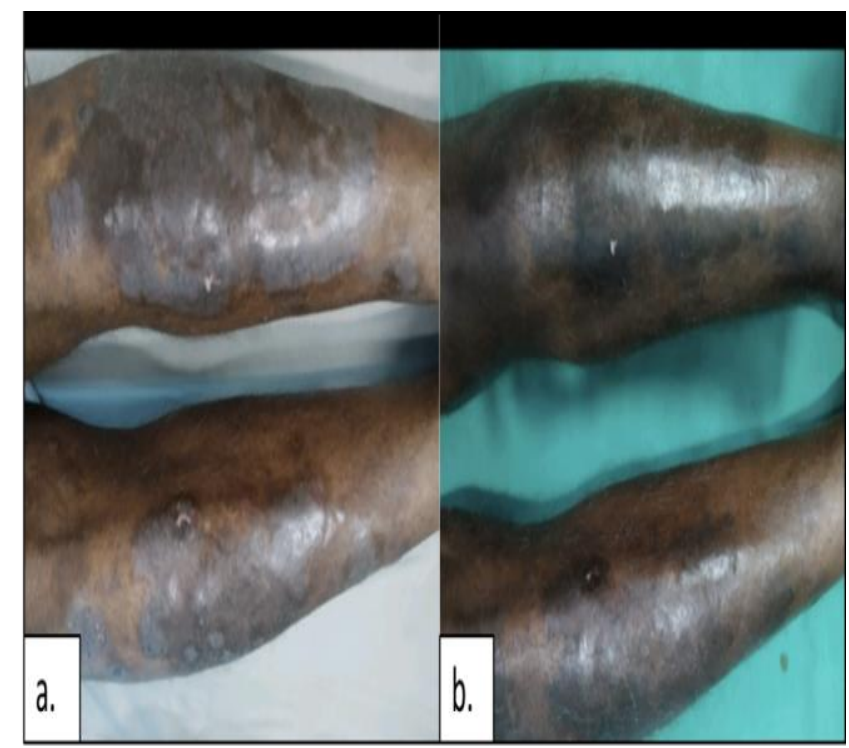

Figure 7 (a and b): Pretreatment image of the lesion on the legs. Post-treatment image of the legs with reduced scaling, erythema, and plaque.

\section{Expert opinion}

Impoyz $^{\mathrm{TM}}(\mathrm{CP})$ cream $0.025 \%$ is associated with lesser local as well as systemic side effects as compared to $\mathrm{CP}$ cream $0.05 \%$. It can be considered as a first-line treatment for managing patients with psoriasis.

\section{Case 8: A case of plaque psoriasis treated with CP $0.025 \%$ for more than 2 weeks}

A 17-year-old male presented with a mildly itchy erythematous scaly plaque over the left leg. There was no significant comorbidity or any family history. The patient's vitals and systemic findings were within normal limits. He had no psychological and social sequelae. The size of the lesions present on the left leg was 2-4 inches (Figure $8 \mathrm{~A})$. Inflammation around the lesions was present. The center of the lesions was elevated. A macular rash was present with scaling and itching. The affected area was warm.

The patient was diagnosed with plaque psoriasis. Topical Impoyz $^{\mathrm{TM}}(\mathrm{CP})$ cream $0.025 \%$ was prescribed to be applied to the affected skin area twice daily for 4 weeks. Additionally, an oral antihistamine, bilastine $20 \mathrm{mg}$ once daily, was prescribed for 2 weeks.

At follow-up, scaling and itching had completely cured. Plaque elevation and erythema had decreased substantially (Figure $8 \mathrm{~B}$ ). Overall, the disease severity had decreased during the treatment period.

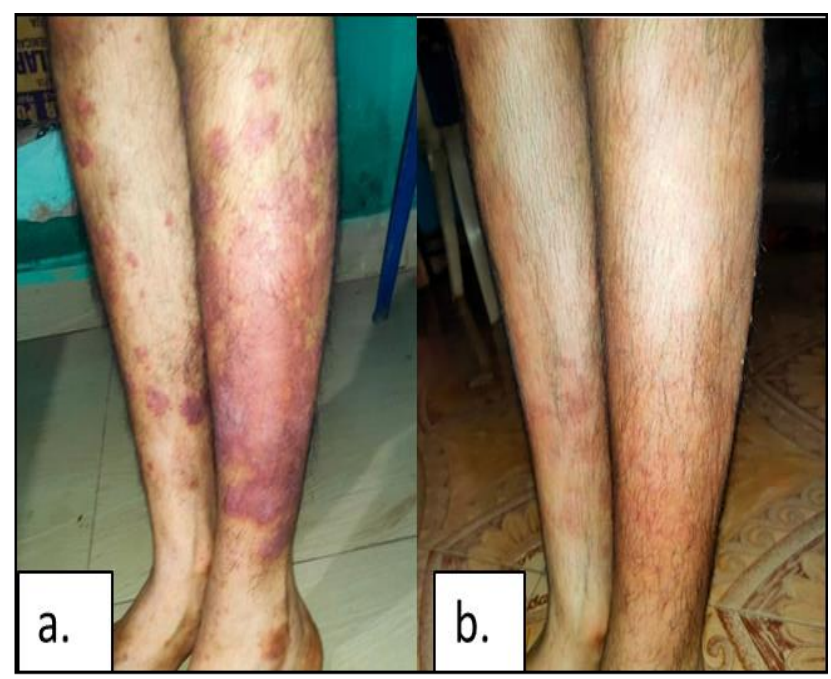

Figure 8 (a and b): Pretreatment image of the lesion on the legs. Post-treatment image of the legs with reduced scaling, erythema, and plaque.

\section{Expert opinion}

Impoyz ${ }^{\mathrm{TM}}(\mathrm{CP})$ cream $0.025 \%$ is an effective treatment for psoriasis. Despite being a potent TC, it is safe for a longer treatment duration as no side effects are seen when used for 4 weeks. 


\section{Case 9: Papular rashes of psoriasis managed effectively with CP $0.025 \%$ cream}

A 14-year-old male presented with an itchy pigmented patch on the right side of the thigh. There was no significant comorbidity or any family history. Physical and systemic examinations showed normal findings, and he showed no psychological and social sequelae. The lesions of around $1 \times 1 \mathrm{~cm}$ were present on the right thigh. Inflammation around the lesions was present. The center of the lesion was scaly. Scaling and itching were present. A papular rash was observed (Figure 9 A).

The patient was diagnosed with psoriasis. The condition was managed with topical Impoyz ${ }^{\mathrm{TM}}(\mathrm{CP})$ cream $0.025 \%$ that was prescribed to be applied to the affected skin area twice daily for 15 days. The cream was advised to be applied after the bath. He was advised to take bath daily with a mild soap.

At follow-up, erythema had decreased substantially (Figure 9 B). The itching had cured completely. Overall, the disease severity had decreased during the treatment period.

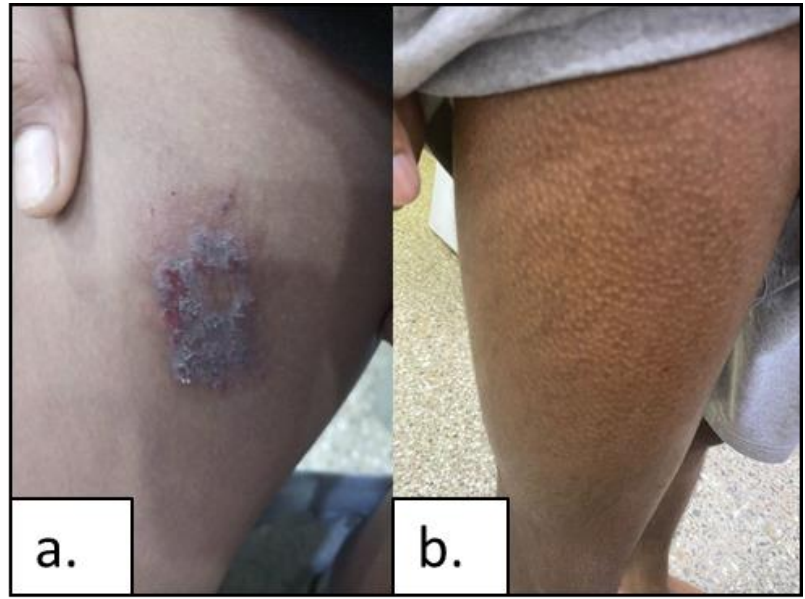

Figure 9 (a and b): Pretreatment image of the lesion on the thigh. Post-treatment image of the thigh with reduced erythema.

\section{Expert opinion}

Impoyz ${ }^{\mathrm{TM}}(\mathrm{CP})$ cream $0.025 \%$ is effective in managing plaque psoriasis. Also, CP cream $0.025 \%$ is relatively safe as compared to other TCs.

\section{Case 10: Role of CP $0.025 \%$ in managing a patient with psoriasis and dyslipidemia}

A 40-year-old female presented with itching and scaling on her hand for the past 2 months. She had high levels of cholesterol and triglycerides for the past 1 year but she was untreated. There was no other comorbidity or any significant family history. She had used detergents for household purposes. Vitals and systemic findings were within normal limits. Psychological and social sequelae were not found.

The lesions of size $2 \mathrm{~cm} \mathrm{x} 3 \mathrm{~cm}$ were present on both hands (Figure $10 \mathrm{~A}$ ). Inflammation was present around the lesions. The center of the lesions appeared thick and scaly. Scaling and itching were present. Crusty skin rashes were seen. The patient was diagnosed with plaque psoriasis.

The patient was prescribed topical Impoyz ${ }^{\mathrm{TM}}(\mathrm{CP})$ cream $0.025 \%$ to be applied to the affected skin areas once daily for 14 days. The cream was advised to be used with occlusion. The patient was advised to avoid using sanitizer.

At follow-up, improvement in scaling and plaque elevation had been noted (Figure $10 \mathrm{~B}$ ). Erythema and itching had reduced substantially. Overall, the disease severity had decreased during the treatment period.

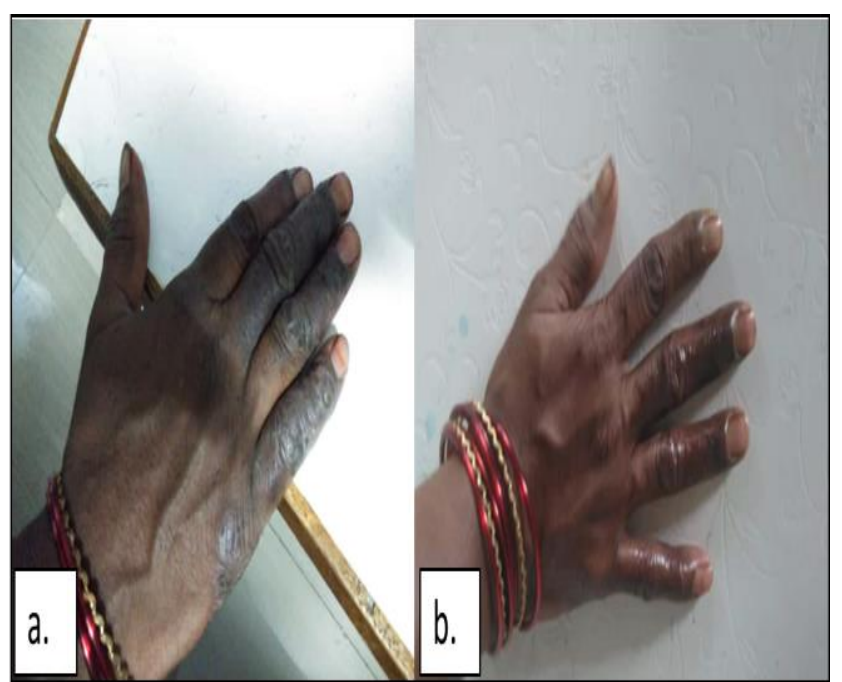

Figure 10 (a and $b$ ): Pretreatment image of the lesion on the dorsal side of the hand. Post-treatment image of the dorsal side of the hand showing improvement.

\section{Expert opinion}

Impoyz ${ }^{\mathrm{TM}}(\mathrm{CP})$ cream $0.025 \%$ is an effective, first-line treatment for plaque psoriasis. CP shows better efficacy compared to other steroids and is relatively safer.

\section{DISCUSSION}

Individuals of no particular age may get affected with psoriasis-a chronic and immune-mediated systemic skin disease. Plaque-type psoriasis is the most common form of psoriasis characterized by erythematous scaly plaques commonly located on scalp, lower back, elbows, umbilical region, intergluteal cleft, and knees. ${ }^{2}$ Plaque psoriasis is an inflammatory disease and warrants the use of an effective anti-inflammatory agent such as $\mathrm{CP} .^{4}$ 
CP has proven efficacy in several chronic skin diseases. It is believed that topical application of $\mathrm{CP}$ can control inflammation and pruritis in atopic dermatitis and psoriasis. ${ }^{3}$ Two crucial randomized controlled trials and a maximal use safety study have established efficacy, skin tolerability, and safety of CP $0.025 \%$ cream. ${ }^{1}$

CP $0.025 \%$ cream formulation is free of common contact allergens and employs diethylene glycol monoethyl ether, which provides increased penetration of active ingredients without their systemic uptake. ${ }^{1}$

A randomized, multicenter, open-label study compared the plasma concentrations of $\mathrm{CP}$ before and after a 2week topical treatment with one of $\mathrm{CP} 0.05 \%$ or $\mathrm{CP}$ $0.025 \%$ formulations. The CP $0.025 \%$ group showed a significantly lower mean serum concentration of $\mathrm{CP}$ compared with the CP $0.05 \%$ group (56.3 vs. 152.5 $\mathrm{pg} / \mathrm{mL}, \mathrm{p}=0.014)$. The results indicate reduced systemic exposure with CP $0.025 \%$ as compared with CP $0.05 \%$ cream, thereby providing a safer treatment of patients with moderate-to-severe plaque psoriasis. ${ }^{5}$

\section{CONCLUSION}

With several new molecules and formulations available for the treatment of various skin conditions, CP $0.025 \%$ shows a clear advantage over others with an improved risk/benefit ratio. It also shows sufficient penetration and better safety profile with respect to side effects than the CP $0.05 \%$ formulation. CP $0.025 \%$ cream is efficacious in reducing clinical signs and symptoms of moderate-tosevere plaque psoriasis. Therefore, CP $0.025 \%$ demonstrates efficacy and safety in psoriasis management and may be considered in patients not candidates for systemic therapy or as an adjunct to systemic therapy.
Funding: No funding sources Conflict of interest: None declared

Ethical approval: Not required

\section{REFERENCES}

1. Del Rosso JQ. Topical corticosteroid therapy for psoriasis-a review of clobetasol propionate $0.025 \%$ cream and the clinical relevance of penetration modification. J Clin Aesthet Dermatol. 2020;13(2):22-9.

2. Uva L, Miguel D, Pinheiro C. Mechanisms of action of topical corticosteroids in psoriasis. Int $\mathbf{J}$ Endocrinol. 2012;2012;561018.

3. Zampetti A, Feliciani C, Tulli A. Pharmacotherapy of inflammatory and pruritic manifestations of corticosteroid-responsive dermatoses focus on clobetasol propionate. Clin Med Insights Ther. 2010;2:523-37.

4. Gottlieb AB, Ford RO, Spellman MC. The efficacy and tolerability of clobetasol propionate foam $0.05 \%$ in the treatment of mild to moderate plaque-type psoriasis of non-scalp regions. J Cutan Med Surg. 2003;7(3):185-92.

5. Draelos ZD, Fowler JF, Cornelison R. A randomized, parallel group, open label, multicenter study to assess the potential for adrenal suppression and systemic drug absorption following multiple dosing with clobetasol propionate cream (Impoyz ${ }^{\mathrm{TM}}$ ), $0.025 \%$ versus clobetasol propionate (Temovate®). J Cutan Med Surg. 2018;2(6):410-20.

Cite this article as: Guttedar A, Mishra N, Gupta SK, Mohankar AD, Sancheti K, Srivastava K et al. Treatment with clobetasol propionate $0.025 \%$ topical therapy in various dermatoses. Int J Res Med Sci 2022;10:250-6. 